\title{
EDITORIAL
}

Check for updates

\section{Reaching higher}

As new events such as surfing, skateboarding, sport climbing and karate are added to the programme of the Tokyo Summer Olympics, we ponder how physics can help enhance athletic performance in elite sport.

Physics has had a long relationship with sport. There is a simple reason for it. "Sport is an early adopter," says Steve Haake, Professor of Sports Engineering at Sheffield Hallam University. "If you have a technology you want to try out, sport could be the quickest way of seeing if it works," adds Haake. Indeed, new materials and insights from computational fluid dynamics have long been pushing performance in elite competitive sports. Take surfing as an example. The neoprene wetsuit, which allows surfers to take to cold waters, was invented in 1952 by former Los Alamos physicist Hugh Bradner. Surfboard fin shapes are optimized with fluid dynamics simulations and, in 2019, a wetsuit with graphene lining promising extra warmth came to the market.

Early adoption certainly seems to be the case for graphene. Graphene made it into several lines of sportswear that came to the market in 2018. It is not hard to see why: graphene is strong, thin, flexible and lightweight, plus it has unique thermal properties that enable good temperature control in textiles. But it is not the only interesting material for sportswear. Auxetic materials ${ }^{1}$ become thicker when stretched so they can provide protection and prevent injury if used in sports equipment thanks to their high energy absorption. Other types of metamaterial such as tensegrity structures that have high strength-to-weight ratios and delocalize deformation could be used in sport footwear

Combining new materials with insights from computational fluid dynamics can make for a winning combination. In the 2018 Winter Olympics in Pyeongchang, South Korea, Dominic Parsons from Great Britain won an unexpected bronze medal in skeleton. He used a graphene-enhanced composite sled: adding graphene toughened the carbon fibre and the composite material was optimized to withstand high g forces and low temperatures. Moreover, the sled was aerodynamically optimized for Parsons, an optimization that involved computational fluid dynamics techniques and laser scanning.

Similarly, computational fluid dynamics studies led to the design of Speedo's LZR racer swimsuits, which dramatically reduce drag, enabling athletes to break numerous world records and win many Olympic medals since they were introduced in 2008. In fact, high-tech swimwear had such an impact on competitive swimming that in 2010 new regulations were introduced to

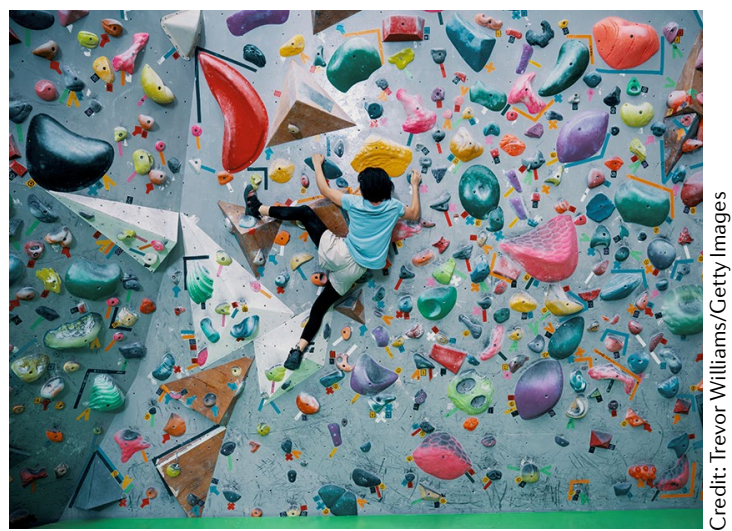

limit the use of high-tech swimwear in competitions ${ }^{2}$. In sports like skeleton or swimming, the smallest boost counts because a difference of a couple of hundredths of a second means a medal or no place on the podium. This makes the pursuit of performance as much a science as a sports competition.

When a new sport joins the Olympic programme, there is usually a boost not only in the pool of athletes, but also in the support they get and the technology they can access. So we will likely soon see new materials in climbing shoes, skateboards and surfboards. Other disciplines, like karate, that do not require special equipment can also benefit from technologies such as sensors and cameras, allowing the measurement of various parameters (athletes' health, training loads and performance). Smart textiles integrating sensors to monitor the athlete's health and performance are the next big thing. But wearable textile sensors enabled by graphene or other 2D materials, for example, are yet to make their way out of the lab.

Whether specializing in 2D materials, metamaterials, fluid dynamics or biophysics, there seem to be interesting sports problems for physicists to address. We hope that, inspired by the Olympics, some of our readers will think of sports as a testing ground for taking physics out of the lab, and in doing so perhaps contribute to future Olympic records.

\footnotetext{
Papadopoulou, A., Laucks, J. \& Tibbits, S. Auxetic materials in design and architecture. Nat. Rev. Mater. 2, 17078 (2017).

2. Foster, L., James, D. $\&$ Haake, S. Influence of full body swimsuits on competitive performance. Procedia Eng. 34, 712-717 (2012).
} 